\title{
Verwertung von Gemüseabfällen - Vergleich der Kompostierung mit dem Verfahren der mikrobiellen Carbonisierung
}

\author{
Erwin Binner · Mathias Egger · Marion Huber-Humer
}

Online publiziert: 25. März 2019

(C) Der/die Autor(en) 2019

Zusammenfassung In 17 Wochen dauernden Praxisversuchen wurden 2 unterschiedliche Verfahren (konventionelle Mietenkompostierung und mikrobielle Carbonisierung) zur Behandlung von Gemüseabfällen getestet. Neben der Frage nach dem für diesen Anwendungsfall besser geeigneten Verfahren interessierte auch der Einfluss von Zwiebeln auf Prozess- und Endproduktqualität. Ein Zwiebelanteil zwischen 0 und $22 \mathrm{Vol}-\%$ hatte bei beiden Verfahren keine negativen Auswirkungen. Hinsichtlich Nährstoffgehalten und Pflanzenverträglichkeit wiesen beide Behandlungsverfahren gleiche Qualität auf. Die konventionelle Kompostmiete lieferte homogenen, krümeligen Kompost mit guter Stabilität (Atmungsaktivität $\mathrm{AT}_{4}<4 \mathrm{mg} \mathrm{O}_{2} / \mathrm{gTM}$, Ammoniumstickstoff $\mathrm{NH}_{4}-\mathrm{N}<25 \mathrm{mg} / \mathrm{kg}$ TM). Bei der mikrobiellen Carbonisierung waren im sehr inhomogenen Endmaterial noch unabgebaute Karotten, Zwiebeln bzw. Pferdemist erkennbar. Das Material war deutlich weniger stabil $\left(\mathrm{AT}_{4}>5\right.$ bis $17 \mathrm{mg} \mathrm{O}_{2} / \mathrm{gTM}$, $\mathrm{NH}_{4}-\mathrm{N} 30$ bis $120 \mathrm{mg} / \mathrm{kg} \mathrm{TM}$ ). Auch die Huminstoffbildung war deutlich geringer verglichen mit dem herkömmlichen Kompostierungsverfahren. Das Endprodukt der mikrobiellen Carbonisierung kann nicht als Kompost im Sinne der Kompostverordnung (BGBl. II 292 2001) bezeichnet werden. Es handelt sich nicht wie in der Kompostverordnung gefordert um ein gesteuertes, aerobes Verfahren, die Hygienisierung kann verfahrensbedingt (es wird nicht umgesetzt) nicht garantiert werden. Bezüglich Methanemissionen wird auch die Forderung des Standes der Technik

DI E. Binner $(\varangle) \cdot$ DI M. Egger . Univ.-Prof. DI Dr. M. Huber-Humer Department Wasser - Atmosphäre Umwelt, Institut für Abfallwirtschaft, Universität für Bodenkultur Wien, Muthgasse 107, 1190 Wien, Österreich erwin.binner@boku.ac.at (es handelt sich um eine Richtlinie des BMLFUW) nach möglichst emissionsarmen Behandlungsverfahren deutlich verfehlt.

\section{Recycling of vegetable waste in agriculture-Comparison of conventional composting and microbial carbonisation according to Witte}

Abstract 2 different techniques (conventional open windrow composting and microbial carbonisation) suitable for converting vegetable residues into a fertilising product proper for agricultural use were tested. On the one hand it was of interest, which of the 2 techniques is advantageous for this type of material, and on the other hand, the effect of onions on processing and quality of end-products were investigated. Onions with a share of 0 up to $22 \mathrm{Vol}$ $\%$ did not show any negative effect. Under the aspects of nutrient contents and plant germination both techniques showed the same quality. The conventional composting trial showed higher homogeneity, more crumb structure and higher stability (respiration activity $\mathrm{AT}_{4}<4 \mathrm{mg} \mathrm{O} / \mathrm{g} \mathrm{DM}$, Ammonia nitrogen $\mathrm{NH}_{4}-\mathrm{N}<25 \mathrm{mg} / \mathrm{kg} \mathrm{DM}$ ). By microbial carbonisation the end product still showed not degraded carrots, onions and horse manure, inhomogeneous composition, less stability $\left(\mathrm{AT}_{4}>5\right.$ to $17 \mathrm{mg} \mathrm{O}_{2} / \mathrm{g}$ DM, $\mathrm{NH}_{4}-\mathrm{N} 30$ to $120 \mathrm{mg} / \mathrm{kg}$ DM) and lower humification. Microbial carbonisation therefore cannot produce compost according to Austrian compost ordinance (BGBl. II 292 2001). The technique does not fulfil controlled aerobic process requirements as recommended by compost ordinance and sanitisation cannot be guaranteed because of missing turning events. Moreover, the requirement of the state of the art of composting (BMLFUW 2005) - processing with emissions as low as possible-cannot be ful- filled by microbial carbonisation technique.

\section{Einleitung}

In der Landwirtschaft bzw. bei der Verarbeitung landwirtschaftlicher Produkte fallen erhebliche Mengen an biogenen Reststoffen an. Anfang 2018 trat die Erzeugerorganisation Marchfeldgemüse $\mathrm{GmbH}(\mathrm{EOM})$ an das ABF-BOKU heran, um neue Möglichkeiten bei der Entsorgung der anfallenden Gemüsereste untersuchen zu lassen. Bis dato wurden diese teuer entsorgt. Dies führt einerseits $\mathrm{zu}$ negativen Umweltauswirkungen und stellt andererseits eine Verschwendung wertvoller Ressourcen dar. Zukünftig sollen die Landwirte diese Reste kompostieren und durch die Anwendung des erzeugten Bodenverbesserungsmittels auf ihren Feldern den Nährstoffkreislauf schließen und Huminstoffaufbau forcieren.

Die wesentliche Forschungsfrage betraf das anzuwendende Behandlungsverfahren. Neben dem konventionellen Mietenkompostierungsverfahren stand auch die Anwendung des Verfahrens der mikrobiellen Carbonisierung nach Witte (s. a.) zur Diskussion.

Die Kompostierung erfordert eine sorgfältige Materialaufbereitung (Zerkleinern, Befeuchten, Mischen, Homogenisieren) und kontinuierliche Betreuung der Kompostmieten. Das Rottegut ist mehr oder weniger arbeitstäglich $\mathrm{zu}$ kontrollieren: Materialstruktur, Feuchtegehalt, Milieubedingungen und Rottetemperaturen sind wesentliche Kontrollparameter. Werden ungünstige Rottebedingungen erkannt, muss durch technische Maßnahmen (z. B. Umsetzen, Wasserzugabe) möglichst rasch gegengesteuert werden. Das in der Regel in wöchentlichen Intervallen durchzuführende Umsetzen erfordert geeignete Maschinen, Energie und Personal.

Unschwer erkennbarer Vorteil der mikrobiellen Carbonisierung gegen- 
über der Kompostierung ist der wesentlich geringere Betreuungsaufwand. Nach der Aufbereitung (Zerkleinerung, Zugabe von Gülle, Mischen, Homogenisierung und Vorrotte) wird das Material $\mathrm{zu}$ einem Haufen aufgeschüttet und verdichtet. Während der gesamten weiteren Behandlung sind lediglich die Temperatur und der Feuchtegehalt zu kontrollieren und letzterer gegebenenfalls durch „Beregnung“ zu optimieren.

Ziel des gegenständlichen Projektes war, das „Witteverfahren“ zu evaluieren bzw. zu beurteilen, ob die Qualität des Endproduktes dieses Prozesses jener von herkömmlichen Komposten entspricht. Dazu wurden 2 aus der gleichen Inputmaterialmischung hergestellte Mieten (eine nach dem herkömmlichem Kompostierungsverfahren, die andere nach dem „Witteverfahren“) wissenschaftlich begleitet. Als Inputmaterialien wurden Karotten, Pferdemist, Rasenschnitt (mit hohem Erdanteil) sowie Strauchschnitt und Rindergülle verwendet.

Eine zweite Forschungsfrage betraf die Kompostierbarkeit von Zwiebeln bzw. deren Einfluss auf Rotteprozess und Kompostqualität. Dies wurde durch die Betreuung von 6 weiteren Mieten mit unterschiedlichen Zwiebelanteilen (10 Vol-\%, $18 \mathrm{Vol}-\%$ und $22 \mathrm{Vol}-$ $\%$, jeweils wieder mit dem herkömmlichen Verfahren und jenem von "Witte“) untersucht. Die beiden oben beschriebenen Mieten enthielten keine Zwiebeln und stellten die "Nullvarianten“ dar.

Die Untersuchungen erfolgten im Rahmen einer Masterarbeit. Um den finanziellen Aufwand (die Finanzierung erfolgte mittels FFG-Innovationsschecks) möglichst gering $\mathrm{zu}$ halten, wurden von den 6 ,Zwiebelvarianten“ außer den Monitoringdaten (Rottetemperatur, Mietenporenluftzusammensetzung) nur die Endprodukte vergleichend untersucht.

\section{Behandlungsverfahren}

\subsection{Kompostierung}

Die Kompostverordnung (BGBl. II 292 2001) definiert Kompostierung als „gesteuerte, exotherme biologische Umwandlung abbaubarer organischer $\mathrm{Ma}$ terialien in ein huminstoffreiches Material mit mindestens $20 \%(\mathrm{~m} / \mathrm{m})$ organischer Substanz". Der Stand der Technik der Kompostierung (BMLFUW 2005) präzisiert, dass Kompostierung ein ae- rober Prozess ist: „Ohne ausreichende Versorgung der Rotteorganismen mit Sauerstoff kommt der aerobe Kompostierungsprozess zum Erliegen".

Das Ziel der Kompostierung ist der zügige und verlustarme Abbau der organischen Ursprungssubstanzen und deren Überführen in stabile, pflanzenfreundliche Humussubstanzen. Bei verlustarmem Abbau verbleibt ein möglichst hoher Anteil der organischen Substanz (Kohlenstoffverbindungen) und der Nährstoffe im Endprodukt (Kompost). Die eingesetzte Verfahrenstechnik und die Betriebsführung sind hinsichtlich möglichst geringer Emissionen zu optimieren (BMLFUW 2005).

Grundvoraussetzung für den aeroben Abbau sind Wasser, Nährstoffe (C/N-Verhältnis) und Sauerstoff. Mikroorganismen können Sauerstoff und Nährstoffe nur in gelöster Form aufnehmen. Bei zu geringem Wassergehalt wird daher der Abbau gehemmt bzw. gänzlich eingestellt. Auch ein zu hoher Wassergehalt hat nachteilige Auswirkungen auf den Rotteprozess. In diesem Fall füllt Wasser die Poren und der Gasaustausch (Zufuhr von Sauerstoff, Abtransport von $\mathrm{CO}_{2}$ und anderen Stoffwechselprodukten) wird behindert. Die Folge sind anaerobe Milieubedingungen, die mit erhöhten Emissionen von Methan und Geruchsstoffen einhergehen.

Nährstoffverteilung und Sauerstoffversorgung werden maßgeblich von der Ausgangsmaterialzusammensetzung und -aufbereitung beeinflusst. Wichtig ist die Schaffung von ausreichend freiem Luftporenvolumen, das konvektive Luftströmung erlaubt. Durch Konvektionsströmung wird einerseits sauerstoffreiche Frischluft in das Rottegut ein- und $\mathrm{CO}_{2}$-reiche Abluft bzw. Stoffwechselprodukte ausgetragen. Strukturreicher Baum- und Strauchschnitt, in gewissem Maß auch Stroh, erhöhen aufgrund des hohen Kohlenstoffgehalts einerseits das $\mathrm{C} / \mathrm{N}$-Verhältnis und sorgen andererseits für Strukturstabilität (Luftporenvolumen) des Rotteguts.

Kohlenstoffreiche Komponenten müssen daher in ausreichendem Maße zugemischt werden. Wesentlich bei der Aufbereitung ist das Herstellen einer günstigen Korngröße. Wird der Strauchschnitt zu stark zerkleinert, mangelt es an Luftporenvolumen. Ausgangsmaterialzusammensetzung und -aufbereitung bestimmen auch die Mietendimensionen. Je geringer die Struktur (Porenvolumen), je geringer der Anteil an
Strukturmaterial, desto geringer müssen die Mietenabmessungen gewählt werden. In der Regel werden in der Bioabfallkompostierung Mietenhöhen zwischen 1,5 und 1,8 m eingesetzt.

Durch technische Maßnahmen ist sicherzustellen, dass für die Mikroorganismen günstige Milieubedingungen geschaffen und während des gesamten Rotteprozesses aufrechterhalten werden. Der Rotteprozess ist daher aufmerksam zu beaufsichtigen. Durch Messung von Rottetemperaturen bzw. Mietenporenluftzusammensetzung $\left(\mathrm{O}_{2}\right.$, $\left.\mathrm{CO}_{2}, \mathrm{CH}_{4}\right)$ sowie organoleptische Bewertung (Geruch, Farbe, Beurteilung der Feuchte) können die Milieubedingungen beurteilt werden. Bei ungünstigen Bedingungen (Austrocknen, anaerobe Bereiche, Geruchsemissionen etc.) ist möglichst rasch entsprechend einzugreifen. Die wesentliche Eingriffsmöglichkeit stellt das „Umsetzen“ der Mieten dar. Dazu sollten spezielle Umsetzgeräte verwendet werden. Im Gegensatz zum Radlader erlauben diese eine deutlich bessere Homogenisierung und Auflockerung des Rottegutes. Durch die Auflockerung werden das Porenvolumen und damit die Sauerstoffversorgung (durch intensivere Konvektionsströmung) verbessert. Bei Bedarf kann vor oder während des Umsetzens auch Wasser zugegeben werden.

Der Aufbau stabiler Huminsubstanzen während der biologischen Behandlung (speziell während der Heißrottephase und nicht wie vielfach angenommen erst während der Nachrotte) konnte u.a. von Grassinger (1998) und Heiss-Ziegler (1999) nachgewiesen werden. Der Huminstoffaufbau wird neben den verwendeten Ausgangsmaterialien (Mischung aus mikrobiologisch leicht und schwer verfügbaren Komponenten) von den Milieubedingungen während der Rotte wesentlich beeinflusst. Ausreichende Sauerstoffversorgung (ohne jedoch dabei die Mineralisierung zu stark zu forcieren) und höhere Temperaturen während der Intensivrottephase fördern die Huminstoffbildung. Unter anaeroben Verhältnissen erfolgt kaum Huminstoffaufbau, allerdings können Stoffwechselprodukte des anaeroben Abbaus förderlich wirken (Smidt et al. 2008).

\subsection{Mikrobielle Carbonisierung nach Witte}

Die Theorie der mikrobiellen Carbonisierung wurde von Walter Witte (s.a.) 




Abb. 1 Homogenisierung des Ausgangssubstrats durch Mischung mit dem Umsetzgerät

postuliert. Laut dieser Theorie steht nicht der aerobe Abbau organischer Materialien im Vordergrund, sondern vielmehr die Zersetzung und der Umbau unter reduzierenden Verhältnissen. Mikroorganismen sind nach Witte auf überwiegend anoxische Verhältnisse angewiesen, um Nährstoffe pflanzenverfügbar zu machen.

Das Substrat für den Prozess soll 60 bis $80 \%$ ligninhaltige Materialien (Stroh, Holz, Rinden) und 40 bis $20 \%$ proteinreiche Komponenten (Harn, Klärschlamm, Fruchtfleisch, Biotonnenmaterial) aufweisen. Die Materialen sind möglichst homogen $\mathrm{zu}$ mischen und auf einen Wassergehalt von 35 bis $50 \%$ anzufeuchten. Während einer einige Tage dauernden „Vorrotte“ wird der in den Materialporen enthaltene Sauerstoff rasch verbraucht und es setzt die sogenannte "Mazeration“ (= Auflösung der Zellstrukturen) ein. Dabei erreicht das Zellwachstum der beteiligten Mikroorganismen die exponentielle Phase. Sobald diese erreicht ist, wird das Material nochmals befeuchtet, homogenisiert und anschließend zur eigentlichen Miete (Höhe 2 bis 2,5 m) aufgeschüttet. Ab diesem Zeitpunkt ist sicherzustellen, dass kein weiterer Sauerstoff in die Miete eindringt. Daher wird die Oberfläche mittels Radladerschaufel verdichtet und die Miete in Folge keinesfalls umgesetzt. Wesentlich ist auch, die Mieten nicht abzudecken, da Lichteinwirkung für lithotrophe Mikroorganismen (v.a. Bakterien und Archaeen) wesentlich ist. Nach 8 bis 12 Wochen ist der Prozess der mikrobiellen Carbonisierung abgeschlossen und das Endprodukt kann in der Landwirtschaft eingesetzt werden.
Laut Witte (s.a.) wird Humus nicht durch Mikroorganismentätigkeit ,aufgebaut", sondern ist ein Rückstand eines unvollständigen Abbaues (vor allem von Lignin) unter den speziellen Bedingungen seines Verfahrens. Im Gegensatz zur Kompostierung, bei der laut Witte 88 bis $92 \%$ des Kohlenstoffs durch Mineralisierung verlorengehen, verbleibt bei der mikrobiellen Carbonisierung der Großteil des Kohlenstoffes (wie auch aller Nährstoffe) im Humus. Es wird sogar ein Großteil des durch Mineralisierung gebildeten $\mathrm{CO}_{2}$ und $\mathrm{CH}_{4}$ über Ameisensäurebildung in Huminstoffen fixiert. Damit können bei diesem Verfahren klimarelevante Emissionen und Nährstoffverluste fast gänzlich vermieden werden.

Tab. 1 Analysenergebnisse der untersuchten Ausgangsmaterialproben der Mietenabschnitte A (ohne Zwiebelzumischung) von konventioneller Kompost-Miete (KK) und „mikrobieller Carbonisierungs“-Miete (MC)

\begin{tabular}{|c|c|c|c|c|c|}
\hline \multirow[t]{2}{*}{ Parameter } & \multirow[t]{2}{*}{ Dimension } & \multicolumn{2}{|c|}{$\begin{array}{l}\text { Konventionelle Kompostie- } \\
\text { rung } \\
\text { (KK-Miete) }\end{array}$} & \multicolumn{2}{|c|}{$\begin{array}{l}\text { Mikrobielle } \\
\text { Carbonisierung } \\
\text { (MC-Miete) }\end{array}$} \\
\hline & & Probe 1 & Probe 2 & Probe 1 & Probe 2 \\
\hline Wassergehalt & {$[\% \mathrm{FM}]$} & 45,2 & 49,1 & 52,0 & 49,7 \\
\hline pH-Wert & {$[-]$} & 7,5 & 7,7 & 7,1 & 7,0 \\
\hline Essigsäure & [mg/kg TM] & 240 & 2000 & 3000 & 2200 \\
\hline Propionsäure & [mg/kg TM] & n.n. & 1200 & n.n. & n.n. \\
\hline Isobuttersäure & [mg/kg TM] & 75 & n.n. & n.n. & n.n. \\
\hline Ammoniumstickstoff & [mg/kg TM] & 240 & 270 & 360 & 230 \\
\hline Glühverlust & {$[\% \mathrm{TM}]$} & 71,8 & 69,0 & 61,7 & 68,9 \\
\hline Organ. Kohlenstoff & {$[\% \mathrm{TM}]$} & 37,2 & 36,8 & 31,7 & 36,3 \\
\hline Gesamtstickstoff & {$[\% \mathrm{TM}]$} & 0,94 & 1,03 & 0,92 & 0,90 \\
\hline $\mathrm{C} / \mathrm{N}$ & {$[-]$} & 40 & 36 & 34 & 41 \\
\hline Atmungsaktivität & [mg 02/g TM] & 50,7 & 57,3 & 62,7 & 45,7 \\
\hline
\end{tabular}




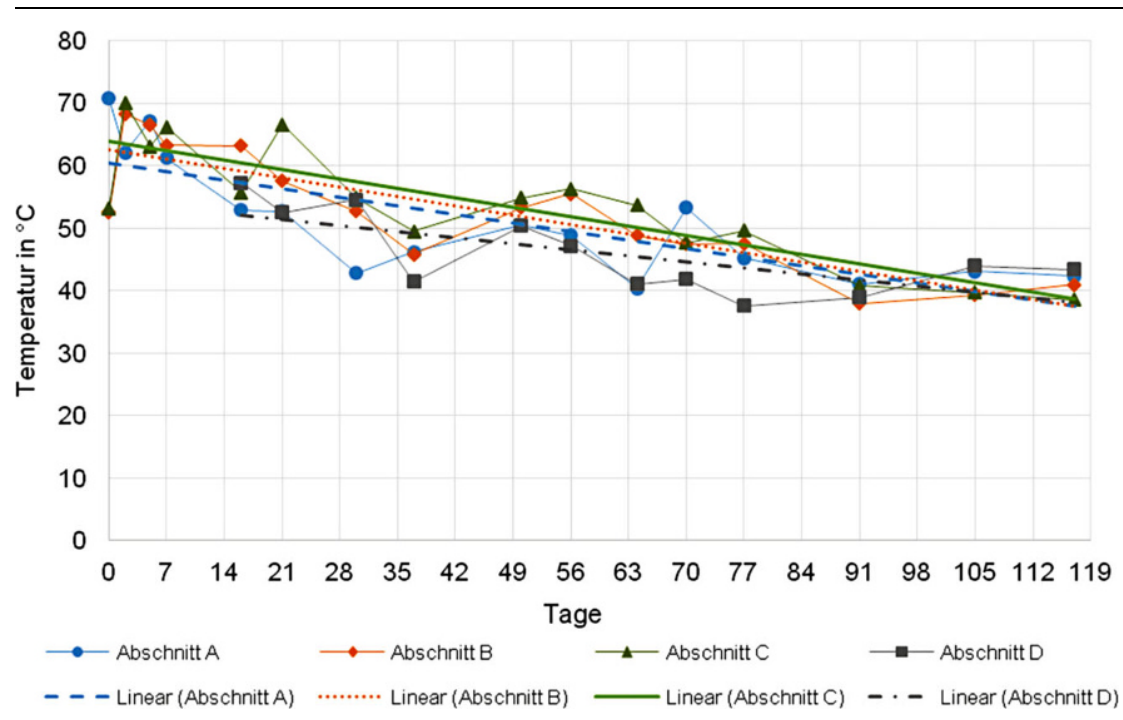

Abb. 2 Verlauf der Rottetemperaturen der 4 Abschnitte (Mittelwert aus jeweils 3 Einzelmessungen) der Kompost-Miete (KK)

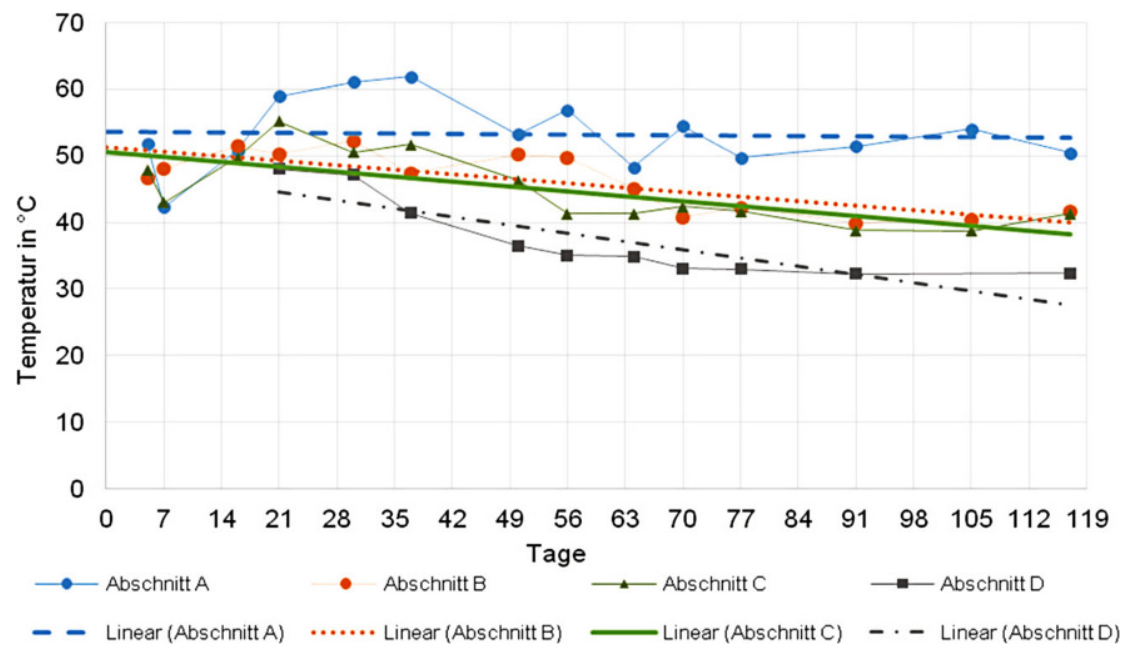

Abb. 3 Verlauf der Rottetemperaturen der 4 Abschnitte (Mittelwert aus jeweils 3 Einzelmessungen) der „mikrobiellen Carbonisierungs“-Miete (MC)

de Verfahren die Mieten unmittelbar nach dem Aufsetzen mit einem traktorgezogenen Kompostwender umgesetzt (Abb. 1).

Nach der verfahrensrelevanten „Vorrotte“ von 3 Tagen wurde die „mikrobielle Carbonisierungs“-Miete nochmals befeuchtet und endgültig aufgesetzt. Danach wurde das Material durch Andrücken mit der Radladerschaufel an der Oberfläche verdichtet. Während des gesamten weiteren Versuchsablaufs wurde die Carbonisierungsmiete wie vorgeschrieben nicht umgesetzt.

Die Mieten wurden messtechnisch überwacht und in regelmäßigen Zeitabständen beprobt. Mietentemperaturen und Zusammensetzung der Mietenpo-
Die Festproben (ca. $3 \mathrm{~kg}$ je Profil) wurden auf die Parameter Wassergehalt (WG), pH-Wert, Leitfähigkeit, Niedere Carbonsäuren (Elution mit Deionat, Messung mittels Gaschromatographen), Ammoniumstickstoff $\left(\mathrm{NH}_{4}-\mathrm{N}\right)$, Nitratstickstoff $\left(\mathrm{NO}_{3}-\mathrm{N}\right), \mathrm{Ge}-$ samtstickstoff $\left(\mathrm{N}_{\mathrm{ges}}\right)$, organischer Kohlenstoff (TOC), organische Substanz (GV), Atmungsaktivität (AT 4 , ÖNORM S 2027, Teil 4 2012), Huminsäurengehalt (adaptierte Methode nach Danneberg (Gerzabek et al. 1993)) und FTIR-Analyse (ÖNORM S 2125 2008) untersucht. Die Endproben (je Miete 2 Mischproben, bestehend aus jeweils 5 Einzelproben) wurden zusätzlich auf Volumengewicht, Wasserkapazität, Nährstoffe (Gesamtgehalte bzw. pflanzenverfügbare Gehalte) $\mathrm{P}, \mathrm{K}, \mathrm{P}_{\mathrm{CAL}}$, $\mathrm{K}_{\mathrm{CAL}}, \mathrm{Ca}$, und $\mathrm{Mg}$ untersucht. Weiters wurde ein Pflanzenverträglichkeitstest (Kressetest) durchgeführt. Die Analysen erfolgten nach Kompostverordnung. Für Parameter deren Analysenmethoden nicht in der Kompostverordnung beschrieben sind, wurden am ABFBOKU entwickelte bzw. entsprechend adaptierte Methoden verwendet.

Anhand der Mess- und Analysenwerte wurden Milieubedingungen $(\mathrm{pH}-$ Wert, Geruchspotenzial), Rottefortschritt (Reaktivität) sowie Endproduktqualität vergleichend beurteilt.

\section{2 „Zwiebelkompostierung“}

Bei allen Varianten (mit Ausnahme der Abschnitte D) wurde die gleiche Inputzusammensetzung verwendet. Gemischt wurden ca. 43 Vol-\% Grünschnitt, 43 Vol-\% Pferdemist und 14 Vol\% Karotten. Der Zwiebelanteil betrug 0 (Abschnitt A), 10 Vol-\% (Abschnitt B) und 18Vol-\% (Abschnitt C). Bei Abschnitt D wurde versucht, alle in der Erzeugerorganisation anfallenden Gemüsereste mit einem möglichst geringen Anteil an „zuzukaufendem“ Grünschnitt zu verarbeiten (11 Vol-\% Grünschnitt, 44 Vol-\% Pferdemist, 22 Vol-\% Karotten und 22 Vol-\% Zwiebel).

Bei den 6 zusätzlichen Zwiebelmieten erfolgte die Mietenbetreuung bzw. die messtechnische Begleitung analog zu Fragestellung 1. Ausgangs- und Zwischenproben wurden nicht entnommen bzw. analysiert. 10 Mischproben der Endprodukte (2 je „Zwiebelmiete" bzw. 1 der Variante $22 \%$ ) wurden ebenfalls analog zu Fragestellung 1 analysiert. 


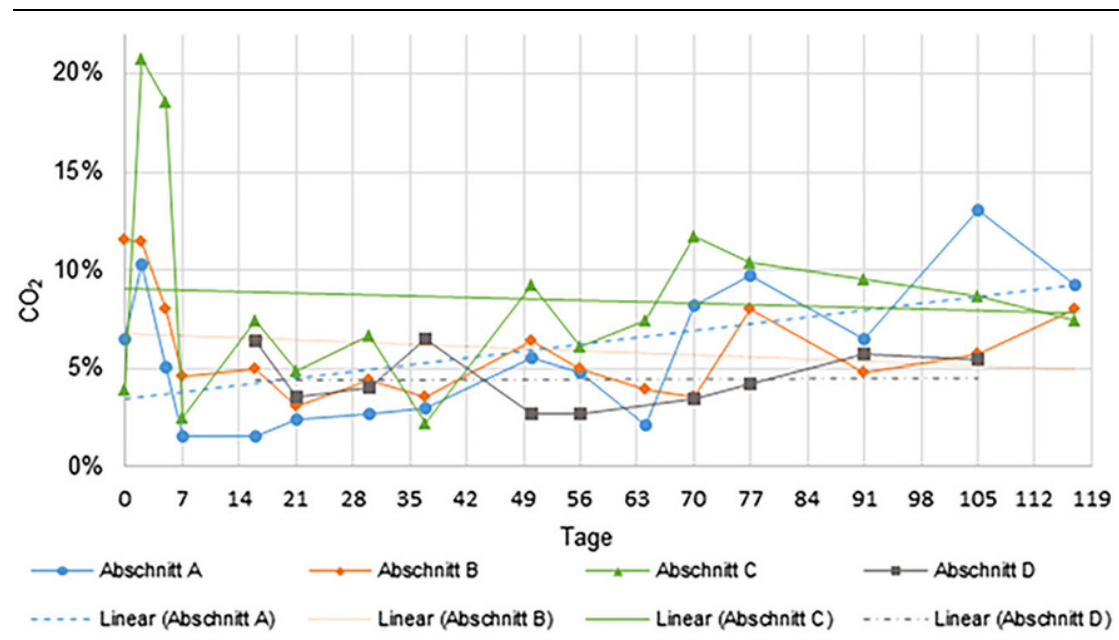

Abb. 4 Verlauf der $\mathrm{CO}_{2}$-Konzentrationen (Vol.-\%) in der Porenluft der 4 Abschnitte (Mittelwert aus jeweils 3 Einzelmessungen) der Kompost-Miete (KK)

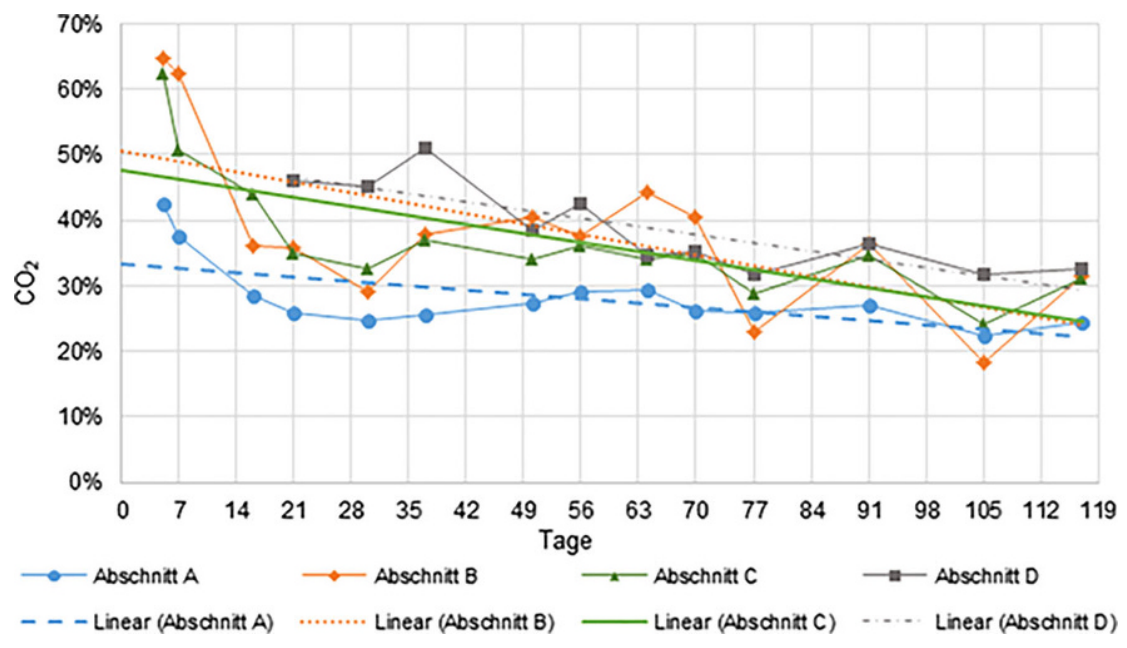

Abb. 5 Verlauf der $\mathrm{CO}_{2}$-Konzentrationen (Vol.-\%) in der Porenluft der 4 Abschnitte (Mittelwert aus jeweils 3 Einzelmessungen) der „mikrobiellen Carbonisierungs“-Miete (MC)

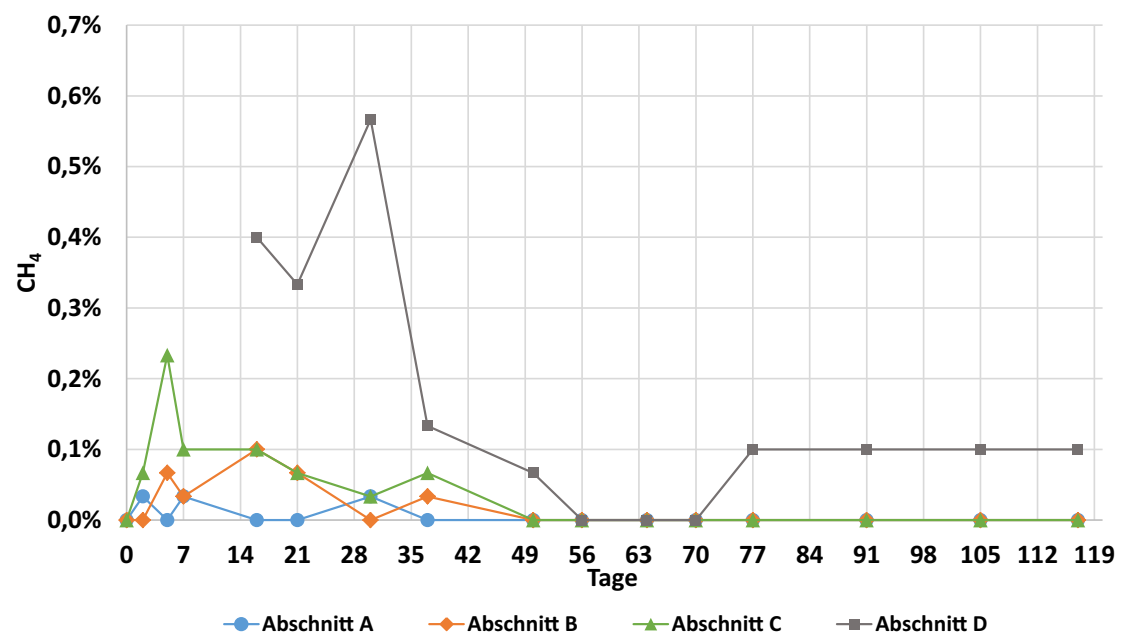

Abb. 6 Verlauf der $\mathrm{CH}_{4}$-Konzentrationen (Vol.-\%) in der Porenluft der 4 Abschnitte (Mittelwert aus jeweils 3 Einzelmessungen) der Kompost-Miete (KK)

\section{Auswertung der Mess- und Analysenergebnisse}

\subsection{Ausgangsmaterialzusammensetzung}

Die Beprobung beider Mieten erfolgte am 18.04.2018, einen Tag nach dem Mischen der Komponenten mittels Umsetzgeräts. Tab. 1 zeigt wesentliche Parameter der Ausgangsmischung in den Bereichen A beider Mieten. Bis zu diesem Zeitpunkt waren beide Mieten gleich behandelt worden, sollten also auch "gleiche“ Analysenergebnisse aufweisen. Die Werte der Tab. 1 zeigen somit die „natürliche“ Schwankungsbreite der hergestellten Mischungen.

\subsection{Prozessbegleitende Untersuchungen}

\subsubsection{Vergleich der Behandlungsverfahren - Rotteverlauf}

Die Rottetemperaturen der konventionellen Kompost-Miete (KK) zeigen in den einzelnen Abschnitten keine wesentlichen Unterschiede (Abb. 2). Über eine Woche lang bleiben die Temperaturen über $60^{\circ} \mathrm{C}$, bis zur 3 . Woche bleiben sie in allen Abschnitten über $50^{\circ} \mathrm{C}$. Eine Hygienisierung des Rotteguts ist damit gewährleistet. Die Trends zeigen mit zunehmender Rottedauer langsam abnehmende Temperaturen.

Bei der „mikrobiellen Carbonisierungs"-Miete (MC) zeigen sich gröBere Unterschiede in den einzelnen Abschnitten (Abb. 3). In Abschnitt A (ohne Zwiebel) bleiben die Temperaturen über den gesamten Rotteverlauf im Bereich 50 bis $60^{\circ} \mathrm{C}$. Die anderen Abschnitte übersteigen kaum die $50^{\circ} \mathrm{C}$ Marke. Trotz der höheren Temperaturen in Abschnitt A ist auch hier die Hygienisierung nicht gewährleistet. Durch den Verzicht auf Umsetzen bleibt das Material der Außenzonen immer in für die Hygienisierung nicht geeigneten Temperaturbereichen. Dies zeigt sich auch durch den dichten Bewuchs der Mietenoberfläche.

Die Porenluftzusammensetzung zeigt bei der KK-Miete (Abb. 4) einen für die Kompostierung typischen Verlauf. Höhere $\mathrm{CO}_{2}$-Konzentrationen treten kurzfristig zu Beginn der Rotte auf. Ab dem 7. Rottetag bewegen sich die $\mathrm{CO}_{2}$ Werte in einem für den aeroben Abbau günstigen Bereich $\left(\mathrm{CO}_{2}<10 \mathrm{Vol}-\%\right)$.

Die $\mathrm{CO}_{2}$-Konzentratonen der MCMiete sind in Abb. 5 dargestellt (man 


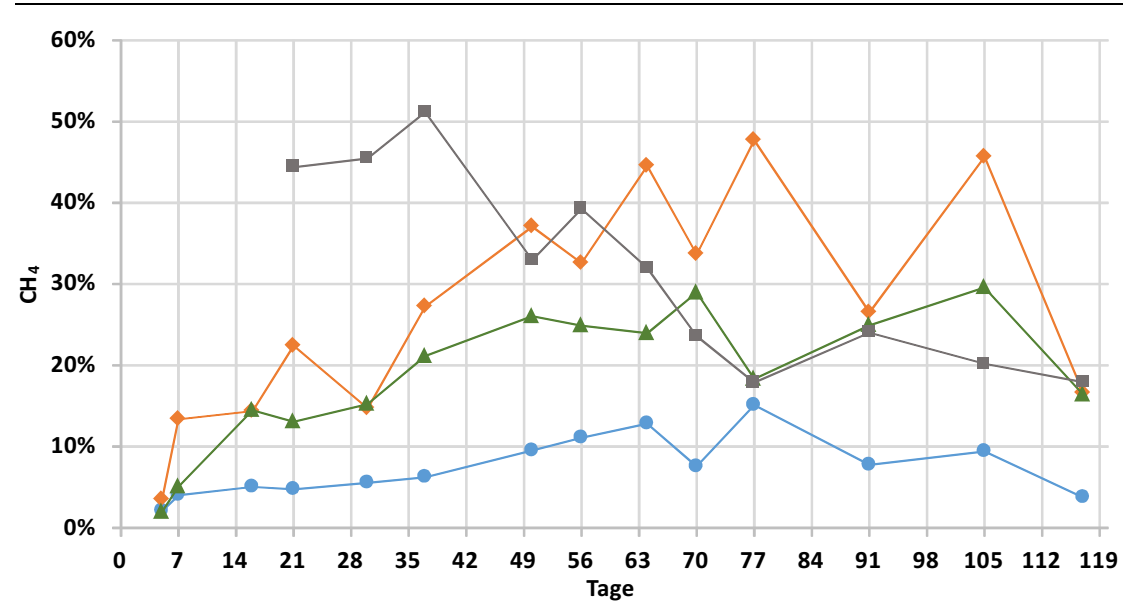

$\rightarrow$-Abschnitt A $\rightarrow$ Abschnitt B $\rightarrow$ Abschnitt C $\rightarrow$ Abschnitt D

Abb. 7 Verlauf der $\mathrm{CH}_{4}$-Konzentrationen (Vol.-\%) in der Porenluft der 4 Abschnitte (Mittelwert aus jeweils 3 Einzelmessungen) er „mikrobiellen Carbonisierungs“--Miete (MC)

\section{Kompost-Miete (KK)}

\begin{tabular}{|c|c|c|c|}
\hline A & B & C & D \\
$\substack{\varnothing 0,73 \mathrm{ppm} \\
( \pm 0,22)}$ & $\begin{array}{c}\varnothing 1,11 \mathrm{ppm} \\
( \pm 0,42)\end{array}$ & $\varnothing 1,09 \mathrm{ppm}$ \\
$( \pm 0,32)$ & $\begin{array}{c}1,79 \mathrm{ppm} \\
( \pm 0,28)\end{array}$ \\
& & \\
\hline
\end{tabular}

\section{„mikrobielle Carbonisierungs“-Miete (MC)}

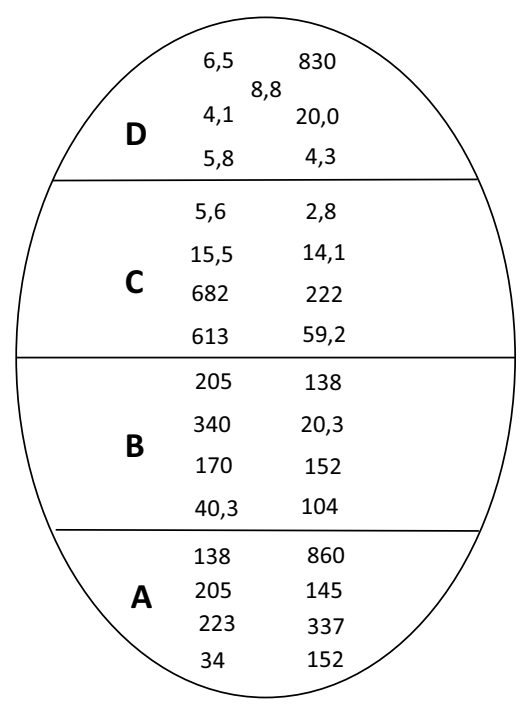

Abb. 8 Ergebnisse der FID-Messungen (Methan in ppm) an den Oberflächen der Mietenabschnitte A bis D der Kompost-Miete (KK, oben) und der „mikrobiellen Carbonisierungs"-Miete (MC, unten) vor Umsetzen und Probenahme beachte die unterschiedliche Skalierung der $\mathrm{y}$-Achse). $\mathrm{Zu}$ Beginn steigen die Werte in allen Abschnitten in einen Bereich zwischen 40 und 60 Vol-\%! Es zeigt sich zwar ein abnehmender Trend, aber auch gegen Ende der Rotte liegen die Konzentrationen noch über $20 \mathrm{Vol}-\%$. Dies weist eindeutig auf anaerobe Milieubedingungen hin.

Bestätigt werden diese Aussagen durch die $\mathrm{CH}_{4}$-Gehalte in der Porenluft. In den Abb. 6 (KK-Miete) und Abb. 7 (MC-Miete) - man beachte wieder die unterschiedliche Skalierung - zeigt die KK-Miete $\mathrm{CH}_{4}$-Konzentrationen unter 0,2 Vol-\% (nur Abschnitt D zeigt an 3 Messtagen Werte bis zu 0,6 Vol-\%). In der MC-Miete zeigen sich (mit Ausnahme von Abschnitt D) steigende Trends von zunächst 10 Vol-\% auf 15 Vol-\% bei Abschnitt A und deutlich höhere Bereiche (40 bis $50 \mathrm{Vol}-\%)$ in den anderen Abschnitten. Die Zwiebelzumischung kann dafür nicht ausschlaggebend sein, weil im Abschnitt mit der höchsten Zumischung eine fallende Tendenz zu beobachten ist, während sich bei den geringeren Zwiebelanteilen ein steigender Trend zeigt.

Die Theorie der mikrobiellen Carbonisierung behauptet, dass Methan und Kohlendioxid die Miete nicht verlassen, sondern in die Huminstoffmatrix eingebaut werden. Dies konnte durch FIDMessungen eindeutig widerlegt werden. Mittels FID (Flammenionisationsdetektor) können auch Spuren von Methan über der Mietenoberfläche detektiert werden. Abb. 8 zeigt die Messergebnisse beider Mieten. Während bei der KK-Miete sehr gleichmäßig niedrige Konzentrationen auftreten (in der Abbildung sind daher nur die Mittelwerte dargestellt) streuen die Ergebnisse bei der MC-Miete sehr stark und liegen wesentlich höher. Die Messwerte liegen, verglichen mit der KK-Miete, bei $155 \%$ bis $47.000 \%$ !

Die im Folgenden beschriebenen Rotteparameter führen alle zur selben Interpretation der Qualität des Rotteprozesses.

Das C/N-Verhältnis wird üblicherweise als Kriterium für den Rottefortschritt betrachtet. Bedingt durch die Mineralisierung von Kohlenstoffverbindungen wird das $\mathrm{C} / \mathrm{N}$-Verhältnis während der Rotte laufend enger (wenn keine Stickstoffverluste auftreten). In der gegenständlichen Untersuchung zeigen sich Unterschiede bei den beiden Varianten (Abb. 9). Der Verlauf der $\mathrm{C} / \mathrm{N}-$ Verhältnisse zeigt zwar bis etwa 


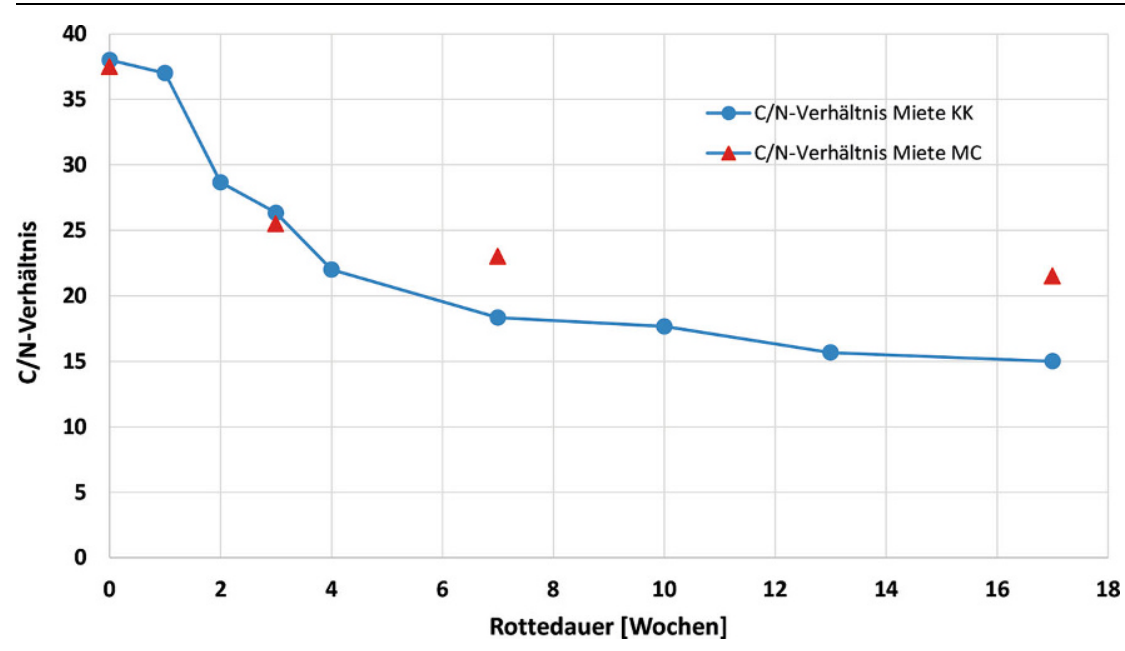

Abb. 9 Verlauf der C/N-Verhältnisse in Kompost-Miete (KK, blaue Kreise) und „,mikrobieller Carbonisierungs"-Miete (MC, rote Dreiecke)

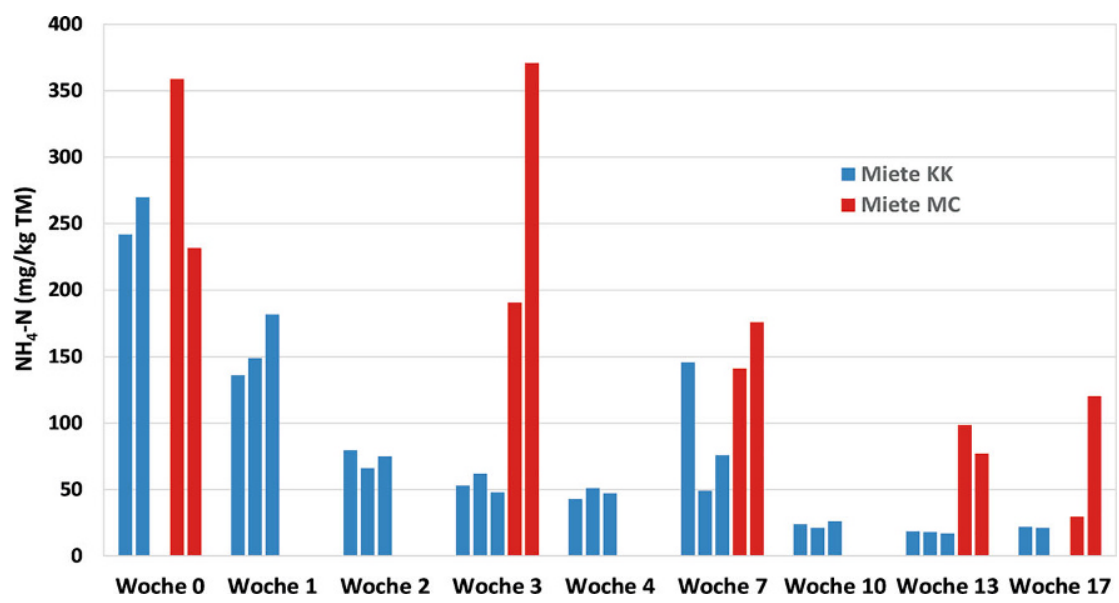

Abb. 10 Verlauf derAmmoniumstickstoffgehalte in Kompost-Miete (KK, blau) und „mikrobieller Carbonisierungs"-Miete (MC, rot)

Tab. 2 Konzentrationen der Niederen Carbonsäuren [mg/kg TM] in den 3 Proben aus Abschnitt A der Kompost-Miete KK (in allen anderen Proben konnten keine Niederen Carbonsäuren nachgewiesen werden)

\begin{tabular}{l|l|l|l|l|l|l|l|l|l|}
\multicolumn{3}{l}{ Essigsäure } & & \multicolumn{3}{l}{ Propionsäure } & & Isobuttersäure \\
Woche & Probe 1 & Probe 2 & Probe 3 & Probe 1 & Probe 2 & Probe 3 & Probe 1 & Probe 2 & Probe 3 \\
\hline 0 & 240 & 2000 & - & n.n. & 1200 & - & 75 & n.n. & - \\
\hline 1 & n.n. & 2500 & 3100 & n.n. & n.n. & n.n. & n.n. & n.n. & n.n. \\
\hline 7 & 550 & 290 & 2900 & n.n. & n.n. & n.n. & n.n. & n.n. & n.n. \\
\hline \multicolumn{7}{l}{ n.n. nicht nachweisbar }
\end{tabular}

Woche 3 in KK und MC parallelen Verlauf. Danach wird das C/N bei der KKMiete deutlich enger als bei der MCMiete.

Die Ammoniumstickstoffgehalte (Abb. 10) bestätigen dieses Bild. Üblicherweise treten zu Beginn der Rotte hohe Konzentrationen auf (bedingt durch den Abbau der organischen Substanz wird $\mathrm{NH}_{4}-\mathrm{N}$ freigesetzt), die bei günstigen Rottebedingungen rasch ab-
Zunahme der Gesamtstickstoffgehalte beweist, dass nicht Stickstoffverluste, sondern die beiden anderen genannten Prozesse dafür verantwortlich sind. Auch dieser Parameter zeigt, dass das Rottegut der MC-Miete nach 17 Rottewochen immer noch nicht ausreichend stabil ist. Die KK-Miete weist deutlich besseren Rottefortschritt auf.

Ungewöhnlich ist allerdings der Anstieg in Rottewoche 7. Offensichtlich kommt es in der KK-Miete zu einem neuerlichen leichten „Abbauschub“. Dadurch werden Ammoniumstickstoff, wie auch Niedere Carbonsäuren (Tab. 2) gebildet.

Carbonsäuren werden bei ausreichender Sauerstoffversorgung des Rottegutes rasch abgebaut. Die MC-Miete zeigt den typischen Verlauf für Sauerstoffunterversorgung (Tab. 3). Sogar nach 17 Rottewochen sind Carbonsäuren noch detektierbar, während in der KK-Miete bereits nach 3 Wochen (mit Ausnahme von Woche 7) Carbonsäuren nicht mehr nachweisbar sind.

Auch die Atmungsaktivität $\left(\mathrm{AT}_{4}\right)$ der MC-Miete zeigt gegenüber der KK-Miete zurückbleibenden Rottefortschritt (Abb. 11). Die größeren Streuungen der Einzelwerte weisen außerdem auf wesentlich inhomogenere Verhältnisse in der MC-Miete hin.

Miete KK zeigt deutlich bessere Huminsäurenbildung als die MC-Miete (Abb. 12). Dies ist nicht weiter verwunderlich, da Huminstoffe nur unter aeroben Bedingungen aufgebaut werden können. Die KK-Komposte zeigen Huminsäurengehalte, die in etwa dem Durchschnitt österreichischer Komposte ( 15 bis $25 \%$ oTM) entsprechen (Smidt et al. 2008). Die MC-Materialien liegen deutlich darunter.

\subsubsection{Vergleich der Behandlungsverfahren - Endproduktqualität}

Am 14.08.2018 erfolgte die Entnahme der Endproben. Die Kompost-Miete (Abb. 13a und c) zeigt keinerlei Bewuchs. Das Rottegut ist sehr homogen, mit Ausnahme von Abschnitt D (hier sind noch einige, wenige Zwiebel zu sehen) sind keinerlei Gemüsereste erkennbar. Die „mikrobielle Carbonisierungs"-Miete (Abb. 13b und d) ist teilweise dicht bewachsen. Zumindest in den oberflächennahen Bereichen wurde somit keine ausreichende Hygienisierung erreicht (Unkrautsamen wurden nicht inaktiviert). Das Material 


\begin{tabular}{|c|c|c|c|c|c|c|}
\hline \multirow[b]{2}{*}{ Woche } & \multicolumn{2}{|c|}{ Essigsäure } & \multicolumn{2}{|c|}{ Propionsäure } & \multicolumn{2}{|c|}{ Isobuttersäure } \\
\hline & Probe 1 & Probe 2 & Probe 1 & Probe 2 & Probe 1 & Probe 2 \\
\hline 0 & 3000 & 2200 & n.n. & n.n. & n.n. & n.n. \\
\hline 3 & 3500 & 8000 & 600 & 900 & n.n. & 360 \\
\hline 7 & 2600 & 6000 & 210 & 550 & n.n. & n.n. \\
\hline 13 & 3500 & n.n. & 270 & n.n. & n.n. & n.n. \\
\hline $17-A$ & n.n. & 2600 & n.n. & 230 & n.n. & 80 \\
\hline $17-C$ & 135 & 250 & 70 & n.n. & n.n. & n.n. \\
\hline
\end{tabular}

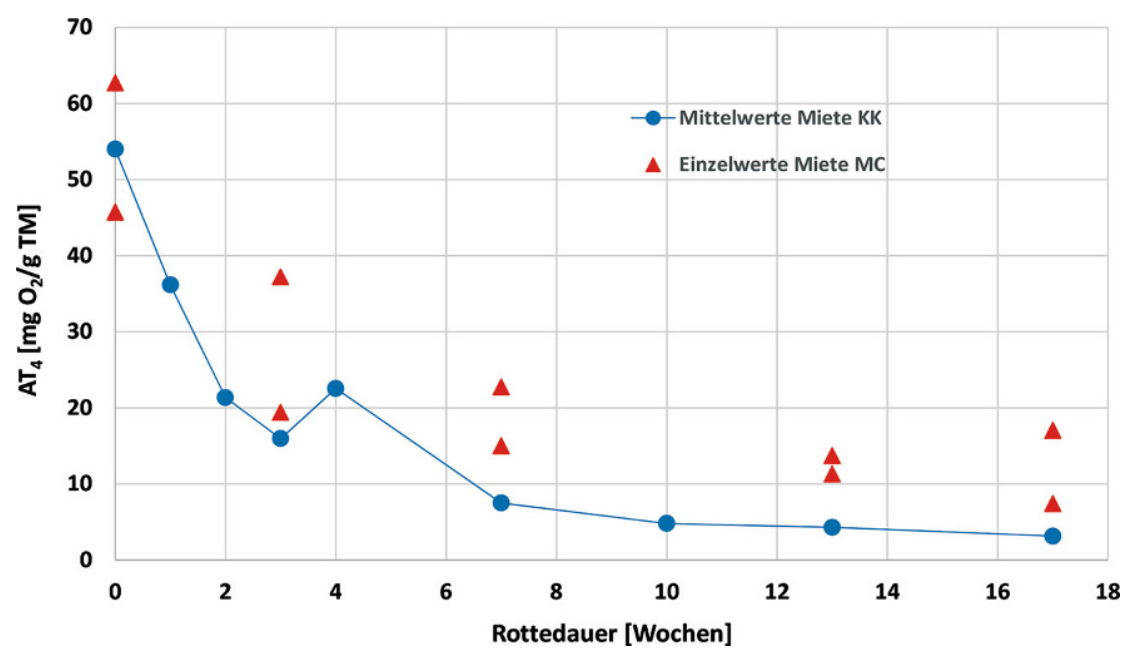

Abb. 11 Verlauf derAtmungsaktivitätin Kompost-Miete(KK, blaue Kreise, Mittelwerte aus je 3 Einzelmessungen) und „mikrobieller Carbonisierungs“-Miete (MC, rote Dreiecke, Einzelwerte)

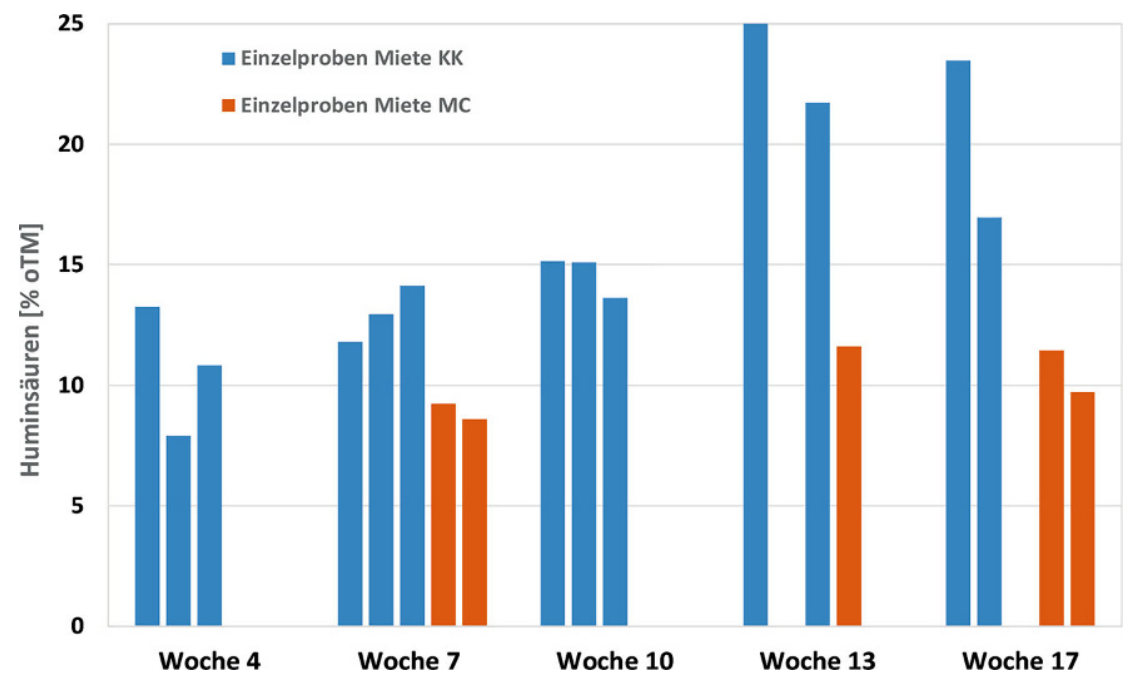

Abb. 12 Verlauf der Huminsäurengehalte [\% oTM] in den Abschnitten A der KompostMiete (KK) und der „mikrobiellen Carbonisierungs“-Miete (MC)
Tab. 4 Ergebnisse der Pflanzenverträglichkeitstests in den $\mathrm{Ab}$ schnitten A, C und D der KompostMiete (KK) und der „mikrobiellen Carbonisierungs"-Miete (MC)

\begin{tabular}{|c|c|c|c|c|}
\hline & \multicolumn{2}{|c|}{$\begin{array}{l}15 \% \text { Substrat- } \\
\text { anteil }\end{array}$} & \multicolumn{2}{|c|}{$\begin{array}{l}30 \% \text { Substrat- } \\
\text { anteil }\end{array}$} \\
\hline & $\begin{array}{l}\text { Keim- } \\
\text { rate } \\
(\%)\end{array}$ & $\begin{array}{l}\text { Bio- } \\
\text { masse } \\
(\%)\end{array}$ & $\begin{array}{l}\text { Keim- } \\
\text { rate } \\
(\%)\end{array}$ & $\begin{array}{l}\text { Bio- } \\
\text { masse } \\
(\%)\end{array}$ \\
\hline \multicolumn{5}{|l|}{ KK-Miete } \\
\hline Abschnitte A & 100 & 100 & 92 & 94 \\
\hline Abschnitt C & 93 & 83 & 100 & 101 \\
\hline Abschnitt D & 100 & 109 & 100 & 108 \\
\hline \multicolumn{5}{|l|}{ MC-Miete } \\
\hline Abschnitte A & 100 & 106 & 100 & 105 \\
\hline Abschnitt C & 100 & 94 & 100 & 103 \\
\hline Abschnitt D & 100 & 107 & 70 & 61 \\
\hline
\end{tabular}

wirkt inhomogen, in allen Abschnitten der Miete ist noch unzersetztes Material (Karotten, Zwiebeln, Pferdemist) erkennbar.

Die Ergebnisse der Kressetests (Tab. 4) zeigen - mit Ausnahme von Abschnitt D bei $30 \%$ Substratanteil in beiden Mieten gleich gute Ergebnisse (die Kompostverordnung fordert bei $15 \%$ Substratanteil $100 \%$ Biomasse, bei $30 \%$ sind $>90 \%$ erforderlich). In keiner der beiden Verfahren sind (mit Ausnahme von Abschnitt D der MC-Miete) nach 17 Wochen Behandlungsdauer toxische Effekte erkennbar.

Die Nährstoffgehalte (Tab. 5) sind bei beiden Mieten von vergleichbarer Qualität. Die MC-Miete weist zwar etwas geringere Konzentrationen auf als die KK-Miete, dies ist aber auf die geringere Mineralisierung zurückzuführen. Durch Mineralisierung kommt es (wegen des Massenverlusts durch Abbau) zu einer „Anreicherung“ der Nährstoffe, vorausgesetzt Verluste (z.B. über Sickerwasseraustrag) können vermieden werden. Würde also die MC-Miete den gleichen Abbaufortschritt wie die KK-Miete aufweisen, wären auch die Nährstoffkonzentrationen im Endprodukt in gleicher Größenordnung. Die Analysenergebnisse widerlegen jedoch die Theorie der mikrobiellen Carbonisierung auch hinsichtlich der Nährstoffgehalte. Danach sollte es durch die MC-Technik zu deutlich höheren Nährstoffkonzentrationen im Endprodukt kommen. 

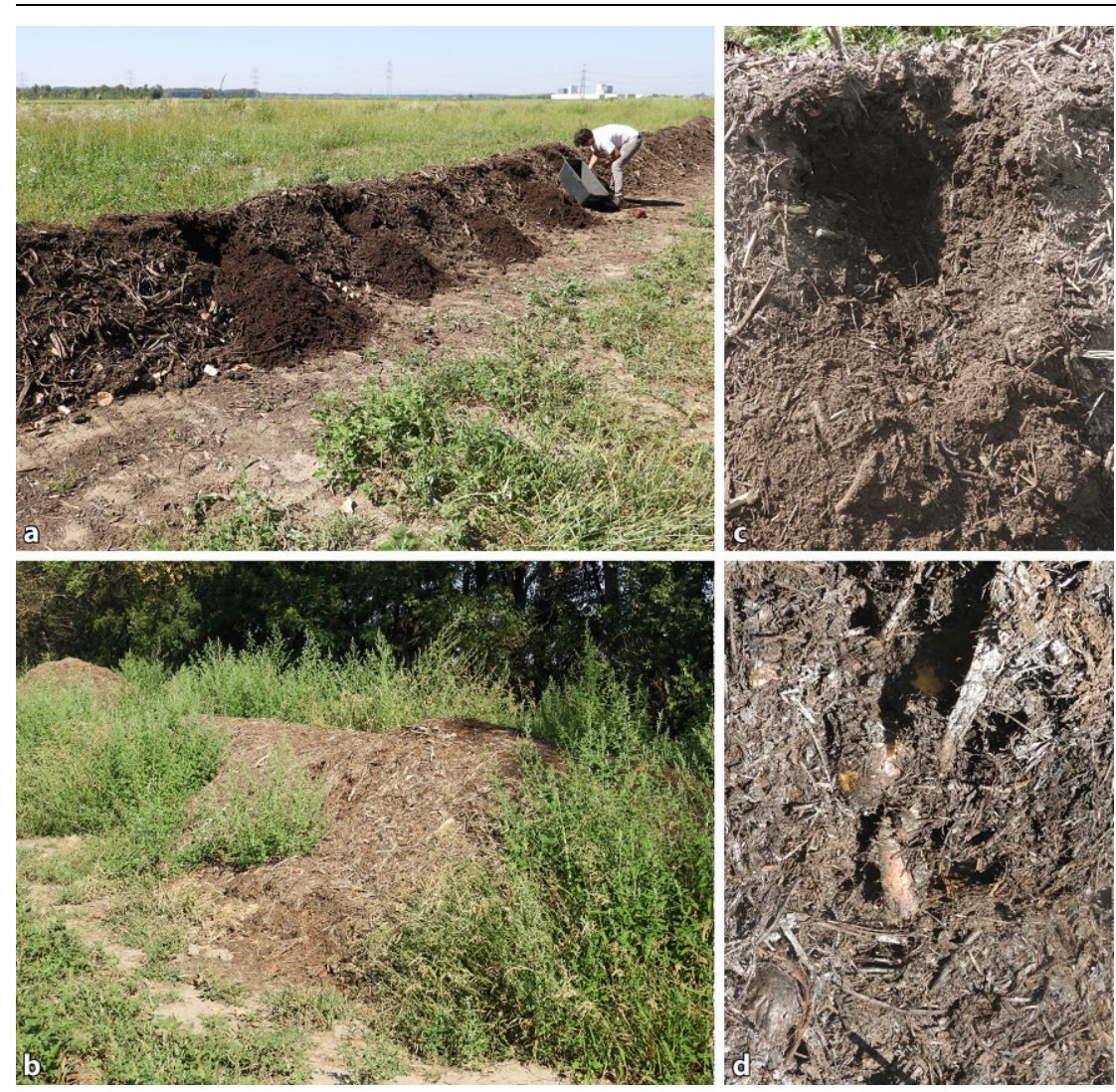

Abb. 13 EntnahmederEndproben:Kompost-Miete (a,c) und „mikrobielleCarbonisierungs"-Miete (b, d)

\subsubsection{Einfluss der Zwiebelzumischung auf Rotteprozess und Kompostqualität}

Die Ergebnisse der gegenständlichen Untersuchung lassen keinen negativen Einfluss der Zwiebel auf Rotteprozess und Endproduktqualität erkennen. Rottetemperaturen (Abb. 4) und Porenluftzusammensetzung (Abb. 6 und 8) in der KK-Miete weisen bei allen Zwiebelanteilen (Abschnitt A bis D) ähnliche Rottebedingungen auf. Sauerstoffversorgung und Hygienisierung sind gewährleistet. Auch in der MC-Miete ist kein Einfluss der Zwiebel erkennbar (Abb. 5, 7 und 9).

Die Ammoniumstickstoffgehalte der Endproben in der gut mit Sauerstoff versorgten KK-Miete sind in allen Abschnitten ähnlich niedrig (Abb. 14). Deutlich höher (wegen des Sauerstoffmangels) sind sie in der MC-Miete. Ein gleiches Bild zeigen die Atmungsaktivitätswerte (Abb. 15). Alle 4 Abschnitte der KK-Miete weisen unabhängig vom Zwiebelanteil gute Stabilität auf. Die Huminsäurengehalte (Abb. 16) in den 4 Abschnitten der KK-Miete liegen zwi- schen 15 und $20 \%$ oTM (eine Probe in Abschnitt A liegt über $20 \%$ oTM). Die Abschnitte der MC-Miete weisen mit 8 bis $15 \%$ oTM geringere Huminsäurenbildung auf. Aber auch hier ist kein Einfluss des Zwiebelanteils erkennbar.

\section{Zusammenfassung}

Im Auftrag der Erzeugerorganisation Marchfeldgemüse $\mathrm{GmbH}$ (EOM) wur-

Tab. 5 Nährstoffgehalte in den Endprodukten der Abschnitte A bis D der KompostMiete (KK) und der „mikrobiellen Carbonisierungs“-Miete (MC)

\begin{tabular}{|c|c|c|c|c|c|c|}
\hline & $\begin{array}{l}\mathrm{P}_{2} \mathrm{O}_{5} \\
\text { (verfügb.) } \\
{[\% \mathrm{TM}]}\end{array}$ & $\begin{array}{l}\mathrm{P}_{2} \mathrm{O}_{5} \\
\text { (gesamt) } \\
{[\% \text { TM] }}\end{array}$ & $\begin{array}{l}\mathrm{K}_{2} 0 \\
\text { (verfügb.) } \\
{[\% \mathrm{TM}]}\end{array}$ & $\begin{array}{l}\mathrm{K}_{2} \mathrm{O} \\
\text { (gesamt) } \\
{[\% \mathrm{TM}]}\end{array}$ & $\begin{array}{l}\text { Mg0 } \\
\text { (gesamt) } \\
{[\% \text { TM] }}\end{array}$ & $\begin{array}{l}\mathrm{Ca0} \\
\text { (gesamt) } \\
{[\% \mathrm{TM}]}\end{array}$ \\
\hline \multicolumn{7}{|l|}{ KK-Miete } \\
\hline Abschnitt A & 0,24 & 0,61 & 1,14 & 1,75 & 1,82 & 5,38 \\
\hline Abschnitt B & 0,19 & 0,53 & 0,84 & 1,39 & 1,91 & 5,52 \\
\hline Abschnitt C & 0,23 & 0,53 & 0,96 & 1,33 & 2,16 & 5,73 \\
\hline Abschnitt D & 0,28 & 0,62 & 1,07 & 1,45 & 2,16 & 5,73 \\
\hline \multicolumn{7}{|l|}{ MC-Miete } \\
\hline Abschnitt A & 0,17 & 0,47 & 0,86 & 1,39 & 1,82 & 4,97 \\
\hline Abschnitt B & 0,11 & 0,38 & 0,55 & 1,14 & 2,40 & 5,59 \\
\hline Abschnitt C & 0,14 & 0,44 & 0,65 & 0,96 & 2,16 & 5,59 \\
\hline Abschnitt D & 0,18 & 0,46 & 0,60 & 0,84 & 1,99 & 4,90 \\
\hline
\end{tabular}

den Praxisversuche zur biologischen Behandlung von Gemüseresten durchgeführt. Im Rahmen einer Masterarbeit am ABF-BOKU wurden 2 Verfahren vergleichend untersucht. Einerseits eine herkömmliche Feldrandmietenkompostierung (Zeilenmiete KK, mit bedarfsgerechter Befeuchtung, regelmäßigem Umsetzen mit gezogener Wendemaschine, 17 Wochen Rottedauer) und andererseits eine Miete nach dem Verfahren der mikrobiellen Carbonisierung (MC) nach Witte („Stapelmiete“, ohne Umsetzen, Behandlungsdauer 17 Wochen). Eine weitere Fragestellung betraf die Mitverarbeitung von Zwiebeln bzw. deren Einfluss auf den Rotteprozess und die Kompostqualität.

Beide Varianten wurden mit den gleichen Inputmaterialien durchgeführt. Verwendet wurden ca. 43 Vol-\% Grünschnitt, 43 Vol-\% Pferdemist und 14 Vol-\% Karotten. Der Zwiebelanteil betrug 0 (Abschnitt A), 10 Vol-\% (Abschnitt B) und 18 Vol-\% (Abschnitt C). Bei Abschnitt D wurde versucht, die anfallenden Gemüsereste mit einem möglichst geringen Anteil an ,zuzukaufendem" Grünschnitt zu verarbeiten (11 Vol-\% Grünschnitt, 44 Vol-\% Pferdemist, 22 Vol-\% Karotten und 22 Vol-\% Zwiebel).

Die Zwiebelzumischung zeigt keinen negativen Einfluss auf Rotteverhalten und Endproduktqualität. Selbst in Abschnitt D mit sehr geringem Strukturanteil konnte in der KK-Variante eine ausreichende Sauerstoffversorgung sichergestellt werden. Bei der MC-Miete zeigt dieser Abschnitt bedingt durch das geringere Kohlenstoffangebot etwas niedrigere Temperaturen und bedingt durch das fehlende Umsetzen höhere $\mathrm{CO}_{2}$ - und $\mathrm{CH}_{4}-$ Konzentrationen in der 


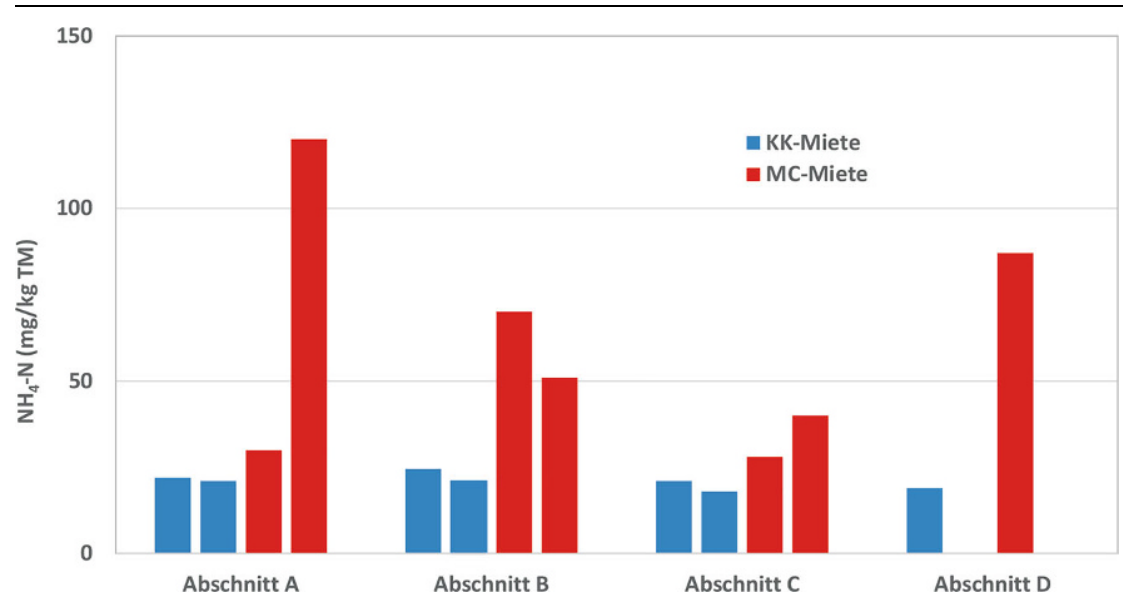

Abb. 14 Ammoniumstickstoff [mg/kg TM] in den Endproben aus Abschnitt A bis D der Kompost-Miete (KK) und der „mikrobiellen Carbonisierungs“-Miete (MC)

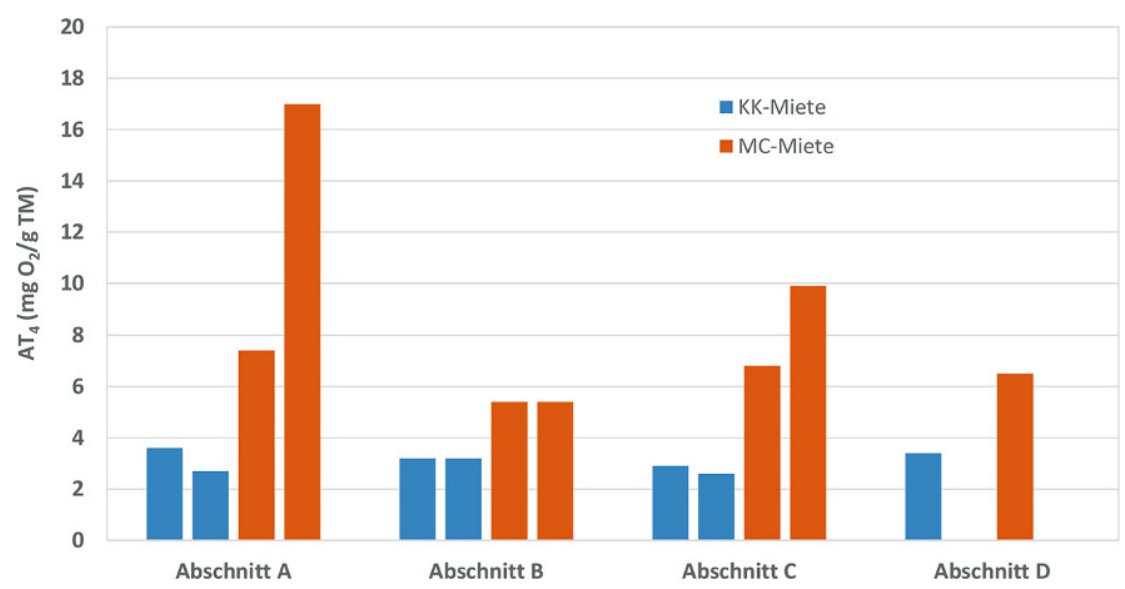

Abb. 15 Atmungsaktivität $\left[\mathrm{mg} \mathrm{O}_{2} / \mathrm{g} \mathrm{TM}\right]$ in den Endproben aus $A$ bschnitt $A$ bis D der Kompost-Miete (KK) und der „mikrobiellen Carbonisierungs“-Miete (MC)

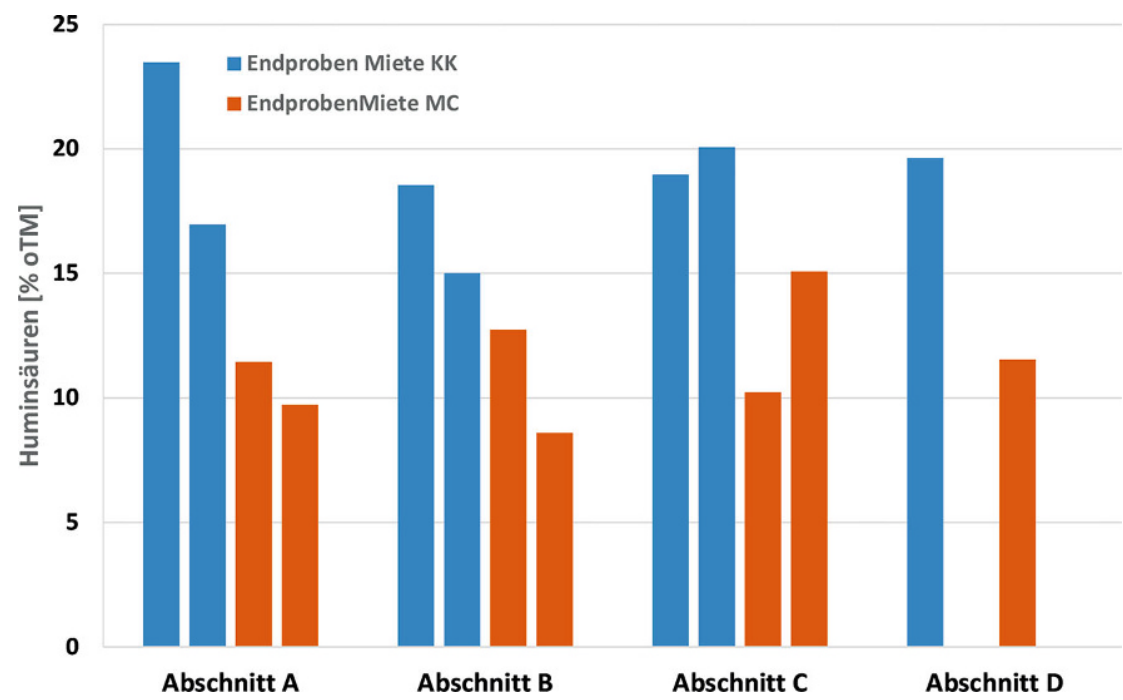

Abb. 16 Huminsäurengehalte [\% oTM] in den Endproben aus Abschnitt A bis D der Kompost-Miete (KK) und der „mikrobiellen Carbonisierungs“-Miete (MC)
Porenluft - also schlechtere Rottebedingungen.

Der Verfahrensvergleich zeigt ganz eindeutig schlechtere Rottebedingungen in der MC-Miete. Sie weist hohe $\mathrm{CO}_{2}$ - und $\mathrm{CH}_{4}$-Konzentrationen in der Porenluft auf, was auch zu höheren Methanemissionen über die Mietenoberfläche führt (belegt durch FIDMessungen). Durch die ungünstigeren Milieubedingungen bleiben die Konzentrationen an Ammoniumstickstoff und Niederen Carbonsäuren höher als in der KK-Variante. Der Huminstoffaufbau (beurteilt anhand der Huminsäurenkonzentrationen) bleibt mit 8 bis $15 \%$ oTM deutlich hinter der KKMiete (15 bis $24 \%$ oTM) zurück. Die Nährstoffgehalte (N, P, K, Ca, Mg) sind in der MC-Miete geringfügig niederer, was auf den gehemmten Abbau der organischen Substanz (Abbau führt zu einer „Anreicherung“ der Nährstoffe durch Masseverlust) zurückzuführen ist. Ein weiteres Indiz für mangelhaften Abbau sind die Analysenwerte von Glühverlust, organischem Kohlenstoff und auch die höhere Atmungsaktivität. Die MC-Miete zeigt in allen Abschnitten noch sichtbare (unzersetzte) Reste von Pferdemist, Karotten und Zwiebeln und weist generell noch gröbere Struktur auf. Hinsichtlich der Pflanzenverträglichkeit zeigen beide Verfahren ähnlich gute Ergebnisse (mit Ausnahme von Abschnitt D der MC-Miete). Ein weiterer Nachteil des MC-Verfahrens ist die mangelnde Hygienisierung, ersichtlich am dichten Bewuchs der Böschungen. Bedingt durch fehlendes Umsetzen erreicht das Material der kühleren Außenzonen nie die für eine Hygienisierung erforderliche Temperatur.

Das Verfahren der mikrobiellen Carbonisierung ist prinzipiell nicht geeignet, Kompost nach Kompostverordnung herzustellen. Die Behandlung beginnt zwar wie gefordert aerob und thermophil, innerhalb kürzester Zeit gleiten die Bedingungen jedoch ins anaerobe Milieu ab. Wegen mangelnder Eingriffsmöglichkeiten kann der Prozess keinesfalls - wie in der Kompostverordnung gefordert - als gesteuert bezeichnet werden. Wegen fehlenden Umsetzens kann die geforderte Hygienisierung (zumindest in den Randzonen) nicht gewährleistet werden.

Emissionsseitig zeigt das Verfahren - zumindest für die untersuchten Materialmischungen - deutlich höhere Emissionen an Methan als das her- 


\section{Originalarbeit}

kömmliche Mietenkompostierungsverfahren. Die Endproduktqualität zeigt hinsichtlich Nährstoffgehalt und Pflanzenverträglichkeit beim MC-Verfahren gleiche Qualität wie bei der Mietenkompostierung. Deutlich schlechter schneidet das MC-Verfahren für die untersuchten Materialmischungen beim Rottegrad (Stabilität, Niedere Carbonsäuren, Ammoniumstickstoff) und bei der Huminsäurenbildung ab. Das Endprodukt ist deutlich inhomogener als jenes der Vergleichsmiete und weist noch beträchtliche Anteile an „unab- gebautem“ Material (Karotten, Zwiebel, Pferdemist) auf.

Funding Open access funding provided by University of Natural Resources and Life Sciences Vienna (BOKU).

Open Access Dieser Artikel wird unter der Creative Commons Namensnennung 4.0 International Lizenz (http:// creativecommons.org/licenses/by/4. $0 /$ deed.de) veröffentlicht, welche die Nutzung, Vervielfältigung, Bearbeitung, Verbreitung und Wiedergabe in jeg- lichem Medium und Format erlaubt, sofern Sie den/die ursprünglichen $\mathrm{Au}$ tor(en) und die Quelle ordnungsgemäß nennen, einen Link zur Creative Commons Lizenz beifügen und angeben, ob Änderungen vorgenommen wurden.

Hinweis des Verlags Der Verlag bleibt in Hinblick auf geografische Zuordnungen und Gebietsbezeichnungen in veröffentlichten Karten und Institutsadressen neutral.

\section{Literatur}

BGBI. II 292 (2001): Verordnung des Bundesministeriums für Land- und Forstwirtschaft, Umwelt und Wasserwirtschaft über Qualitätsanforderungen an Komposte aus Abfällen (Kompostverordnung)

BMLFUW (2005): Stand der Technik der Kompostierung. Richtlinie des Bundesministeriums für Land- und Forstwirtschaft, Umwelt und Wasserwirtschaft

Gerzabek, M. H., Danneberg, O. und Kandeler, E. (1993): Bestimmung des Humifizierungsgrades, Bodenbiologische Arbeitsmethoden, Springer Verlag, Wien, S. 107-109
Grassinger, D. (1998): Einfluss von Temperatur und Sauerstoffgehalt auf die Humifizierung und die Mineralisierung bei der Verrottung von Bioabfall. Dissertation am ABF-BOKU

Heiss-Ziegler, C. (1999): Verhalten von Stickstoff bei der Ablagerung von Abfällen. Dissertation am ABF-BOKU

ÖNORM S 2027-4 (2012): Beurteilung von Abfällen aus der mechanisch-biologischen Behandlung - Teil 4: Stabilitätsparameter - Atmungsaktivität (AT4)

ÖNORM S 2125 (2008): FTIR-Messung von Abfällen
Smidt, E.. Binner, E., Meissl, K., Tintner, J., Lechner, P. (2008): Huminstoffe als Qualitätsparameter für Komposte und zur verfahrenstechnischen Optimierung von Kompostanlagen Forschungsbericht gefördert von der FFG (Projekt-Nr. 808753 / 812303 / 814375)

Witte, W. (s. a.): Die Microbielle Carbonisierung Teil 1. Hrsg: Walter Witte 